\title{
Doxapram shortens recovery following sevoflurane anesthesia
}

\author{
[Le doxapram hâte la récupération après une anesthésie au sévoflurane] \\ Chi-Chen Wu MD, ${ }^{*}$ Martin S. Mok MD, ${ }^{*}$ Jui-Yuan Chen MD, $†$ Gong-Jhe Wu MD, $†$ Yeong-Ray Wen MD,$\dagger$ \\ Chao-Shun Lin MD*
}

Purpose: A randomized, double blind controlled trial was undertaken to investigate the effect of doxapram on recovery times and bispectral index following sevoflurane anesthesia.

Methods: Upon completion of surgery under sevoflurane anesthesia, 60 adult patients were randomly allocated to receive either doxapram hydrochloride I $\mathrm{mg} \cdot \mathrm{kg}^{-1}$ iv or saline placebo. Clinical recovery from anesthesia was assessed by time to eye opening on verbal command, hand squeezing on command, time to extubation, and the Aldrete recovery score. Bispectral index values, systolic blood pressure, and heart rate were recorded at baseline (before anesthesia), during surgery, and every minute for 15 min after administration of the study drug. Results: Time to eye opening was shorter in the doxapram group compared with the control group $(6.9 \pm 2.2 \mathrm{~min}$ vs $9.9 \pm 3.1 \mathrm{~min}, P<0.05)$. Mean bispectral index scores were also higher in the doxapram group compared with the saline placebo seven to eight minutes following administration of the study medication $(P<0.05)$. More rapid emergence was associated with a greater increase in heart rate with doxapram $(P$ $<0.05$ compared with placebo), but no differences in systolic blood pressure responses were observed in comparison with placebo.

Conclusion: We conclude that doxapram I mg.kg-1 hastens early recovery from sevoflurane anesthesia, and this arousal effect correlates with higher bispectral index values.

Objectif : Examiner l'effet du doxapram sur les temps de récupération et l'index bispectral à la suite d'une anesthésie au sévoflurane par une étude randomisée, contrôlée et à double insu.

Méthode : L'opération complétée sous anesthésie au sévoflurane, 60 patients adultes répartis au hasard ont reçu $I \mathrm{mg} \cdot \mathrm{kg}^{-1}$ de chlorhydrate de doxapram iv ou un placebo de solution salée. La récupération clinique de l'anesthésie a été évaluée par le moment de l'ouverture des yeux ou de la réaction à une commande verbale, la possibilité de serrer la main sur demande, le temps d'extubation et le score de récupération d'Aldrete. Les valeurs de l'index bispectral, la tension artérielle systolique et la fréquence cardiaque ont été enregistrées avant l'anesthésie, pendant l'opération et à chaque minute pendant 15 min après l'administration du médicament.

Résultats : Le temps écoulé avant l'ouverture des yeux a été plus court avec le doxapram qu'avec le placebo (6,9 $\pm 2,2$ min vs 9,9 $\pm 3, I \mathrm{~min}, P<0,05)$. Les scores moyens de l'index bispectral ont été aussi plus élevés avec le doxapram sept à huit minutes après l'administration du médicament expérimental $(P<0,05)$. Un retour à la conscience plus rapide a été associé à une plus grande élévation de la fréquence cardiaque avec le doxapram $(P<0,05$ comparé au placebo), mais aucune différence intergroupe de tension artérielle systolique n'a été observée.

Conclusion : Nous concluons que $/ \mathrm{mg} \cdot \mathrm{kg}^{-1}$ de doxapram accélère la récupération après une anesthésie au sévoflurane. Cet effet est corrélé par les valeurs plus élevées de l'index bispectral.

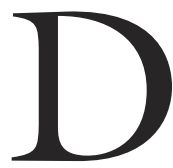
OXAPRAM is a respiratory stimulant with effects on both peripheral and central chemoreceptors. ${ }^{1-5}$ In addition, doxapram is also a central nervous system (CNS) stimulant and has been shown to hasten the recovery from general anesthesia with barbiturates, ether, cyclopropane, halothane and methoxyflurane.$^{6-10}$ However, its effect on sevoflurane, an inhalation anesthetic with a low blood/gas solubility coefficient and rapid recovery, has not been determined. Furthermore, the effect of doxapram on bispectral index (BIS), a widely used clinical monitor for objectively evaluating the hypnotic effect of anesthesia ${ }^{11,12}$ and the early recovery

From the Departments of Anesthesiology, Taipei Medical University Hospital, ${ }^{*}$ and Shin Kong Wu Ho-Su Memorial Hospital, $†$ Taipei, Taiwan.

Address correspondence to: Dr. Chao-Shun Lin, Department of Anesthesiology, Taipei Medical University Hospital, $252 \mathrm{Wu}-\mathrm{Hsin}$ Street,

Taipei, Taiwan 110. Phone: 886-2-27372181, ext. 1212; Fax: 886-2-27367344; E-mail: soon.lin@msa.hinet.net

Accepted for publication November 7, 2005.

Revision accepted December 20, 2005.

Competing interests: None declared. 
phase of sevoflurane anesthesia, is also unknown. The objective of the present study was to investigate the effect of doxapram on early clinical recovery and BIS in patients following sevoflurane anesthesia.

\section{Methods}

Following Hospital Ethics Committee approval of the protocol for this double-blind, randomized prospective study, written informed consent was obtained from 60 adult patients of ASA class I and II physical status. Enrolled patients had no cardiovascular, pulmonary, or neurological diseases, and were scheduled for elective orthopedic surgeries of the lower extremities. No premedication was given. Upon arrival in the operating room, usual monitors were applied to each subject, including a precordial stethoscope, continuous electrocardiogram, peripheral pulse oximeter, non-invasive blood pressure monitor, and an end-tidal $\mathrm{CO}_{2}$ monitor. In addition, after cleansing the skin with alcohol, a disposable BIS electrode was placed on the patient's forehead and connected to the BIS monitor (Ultraview Bispectral Index Module, Aspect Medical System, Natick, MA, USA). The BIS values, signal quality index and electromyograph bar graph were monitored continuously, and recorded at oneminute intervals throughout the study period.

After recording baseline vital signs and BIS values, anesthesia was induced with thiopental $4 \mathrm{mg} \cdot \mathrm{kg}^{-1}$ iv and fentanyl $100 \mu \mathrm{g} i v$. Endotracheal intubation was facilitated with succinylcholine $1 \mathrm{mg} \cdot \mathrm{kg}^{-1} i v$. After intubation the inspiratory and expiratory concentrations of oxygen and sevoflurance were monitored. Anesthesia was maintained with sevoflurane 2-3\% end-tidal concentration with oxygen, titrated to maintain BIS scores in the range of 45-50. Incremental doses of atracurium $0.1 \mathrm{mg} \cdot \mathrm{kg}^{-1}$ in were given as required to maintain adequate muscle relaxation. No additional opioid was used during surgery. During the last $30 \mathrm{~min}$ of the operation no further muscle relaxant was administered, and anesthesia was continued with assisted ventilation to maintain end-tidal $\mathrm{CO}_{2}$ values between $35-40 \mathrm{mmHg}$. Five minutes prior to anticipated completion of surgery, sevoflurane was discontinued and the patient's lungs were ventilated with $100 \%$ oxygen at a fresh gas flow rate of $5 \mathrm{~L} \cdot \mathrm{min}^{-1}$. Guided by a train-of-four neuromuscular blockade monitor (TOF-Guard, Organon Teknika BV, Boxtel, Netherlands) reversal of residual neuromuscular block was achieved with neostigmine $0.05 \mathrm{mg} \cdot \mathrm{kg}^{-1}$ iv and atropine $0.01 \mathrm{mg} \cdot \mathrm{kg}^{-1} i$.

Patients were next randomly divided into two groups of equal size according to a computerized randomization table. The control Group C patients $(n=$
30) received normal saline $i v$, and Group D patients $(n$ = 30) received doxapram $1 \mathrm{mg} \cdot \mathrm{kg}^{-1}$ iv (Nhwa Pharma Corporation, Xuzhou, China) in identically appearing syringes. Study medication or placebo was administered by a dedicated research assistant who was blinded to the syringe contents, which were administered immediately after discontinuation of sevoflurane. Recovery from anesthesia was assessed by a blinded anesthesiologist. The following parameters were evaluated: eye opening on verbal command; hand squeezing in response to verbal command, and time to extubation of the trachea after discontinuation of the anesthetic gas. Heart rate, systolic blood pressure, BIS values, and $\mathrm{SpO}_{2}$ values were determined before, and every five minutes during surgery, then every minute after the injection of the study drugs for $15 \mathrm{~min}$. End-tidal $\mathrm{CO}_{2}$ concentration and end-expiratory concentration of sevoflurane were also recorded from the time of study drug injection to the time of extubation. Aldrete recovery scores ${ }^{13}$ were recorded upon arrival in the postanesthesia care unit (PACU) and at the time of discharge from PACU. Patients were questioned specifically about any recall or awareness during anesthesia or any abnormal psychological feeling during emergence.

The primary end-point of this study was defined as the time to achieve eye opening to verbal command. Applying an a priori power analysis, at least 22 patients had to be enrolled in each treatment group to provide $80 \%$ power to detect a difference at $\alpha=$ 0.05 . Data are expressed as mean and standard deviation. Demographic data and Aldrete scores were analyzed by the Chi-square test. Bispectral index values, end-tidal $\mathrm{CO}_{2}$, end-expiratory sevoflurane concentration, heart rate, and systolic blood pressure were analyzed by repeated-measures analysis of variance (ANOVA) and the Newman-Keuls test was applied when ANOVA was significant. Times to eye opening, response to verbal command, and extubation were also compared using repeated-measures ANOVA. Data are presented as mean \pm standard deviation, and statistical significance was assumed when $P<0.05$.

\section{Results}

All enrolled subjects completed the study protocol. Demographic characteristics were similar between groups (Table I). Groups were also comparable with respect to duration of anesthesia, end-tidal $\mathrm{CO}_{2}$ values, and end-tidal sevoflurane concentrations (Table I). No patient received any medication during the course of anesthesia, which was not specified by the study protocol.

Mean times to eye opening, hand squeeze to command, and extubation of the trachea were signifi- 
TABLE I Demographic and clinical data

\begin{tabular}{lll}
\hline & $\begin{array}{l}\text { Control } \\
(n=30)\end{array}$ & $\begin{array}{l}\text { Doxapram } \\
(n=30)\end{array}$ \\
\hline Age $(\mathrm{yr})$ & $38.6 \pm 9.7$ & $38.8 \pm 11.0$ \\
Gender $(\mathrm{M} / \mathrm{F})$ & $16 / 14$ & $15 / 15$ \\
Body height $(\mathrm{cm})$ & $159.2 \pm 6.7$ & $160.5 \pm 5.3$ \\
Body weight $(\mathrm{kg})$ & $56.6 \pm 9.1$ & $58.9 \pm 12.6$ \\
$\begin{array}{l}\text { Hematocrit }(\%) \\
\text { Anesthesia duration }(\mathrm{min})\end{array}$ & $37.6 \pm 3.5$ & $38.5 \pm 4.3$ \\
$\begin{array}{l}\text { End-tidal } \mathrm{CO}_{2} \text { at tracheal } \\
\text { extubation }(\mathrm{mmHg})\end{array}$ & $169.5 \pm 53.5$ & $166.6 \pm 73.6$ \\
$\begin{array}{l}\text { End-tidal sevoflurane at } \\
\text { tracheal extubation }(\%)\end{array}$ & $35.6 \pm 4.3$ & $36.1 \pm 5.1$ \\
\hline
\end{tabular}

$\mathrm{M}=$ male; $\mathrm{F}=$ female.

TABLE II Recovery parameters

\begin{tabular}{lll}
\hline & $\begin{array}{l}\text { Control } \\
(n=30)\end{array}$ & $\begin{array}{l}\text { Doxapram } \\
(n=30)\end{array}$ \\
\hline Eye opening $(\mathrm{min})$ & $9.9 \pm 3.1$ & $6.9 \pm 2.2^{*}$ \\
Response to command $(\mathrm{min})$ & $10.6 \pm 3.1$ & $7.6 \pm 2.1^{*}$ \\
Extubation of trachea $(\mathrm{min})$ & $11.2 \pm 3.8$ & $8.7 \pm 2.4^{*}$ \\
\hline${ }^{*} P<0.05$ when compared with control group. Value expressed as \\
mean \pm standard deviation.
\end{tabular}

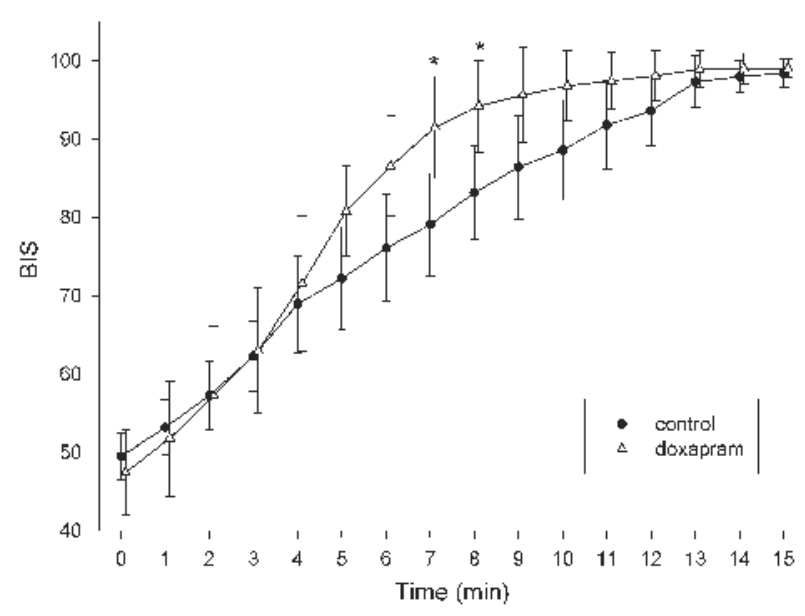

FIGURE 1 Changes in bispectral index (BIS) after injection of study drug. ${ }^{*} P<0.05$ when compared with placebo.

cantly shorter in the doxapram group $(P<0.05)$ in comparison with placebo (Table II). Mean BIS scores were similar in doxapram and control groups prior to administration of the study drug. While BIS scores recovered rapidly upon discontinuation of sevoflurane, BIS scores were significantly higher in the doxapram group compared with placebo seven to eight

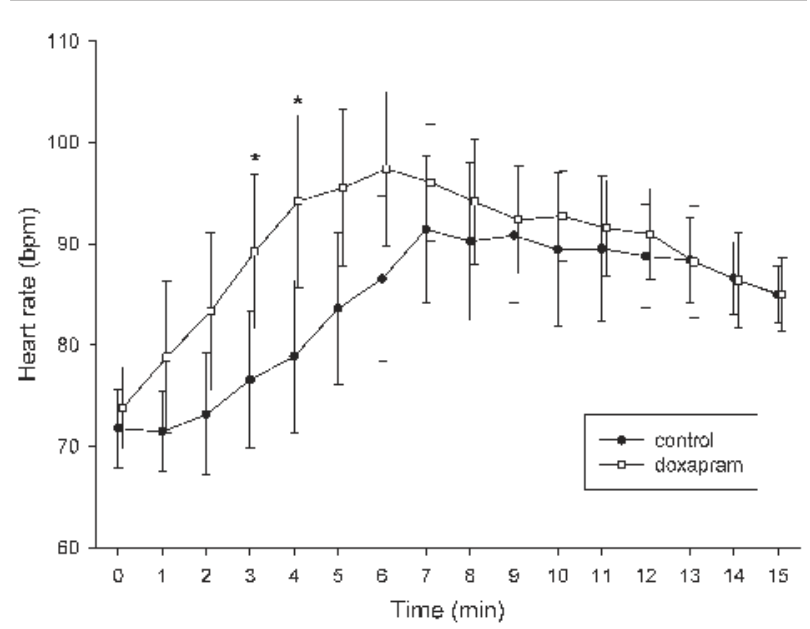

FIGURE 2 Changes in heart rate after injection of study drug. ${ }^{*} P<0.05$ when compared with placebo.

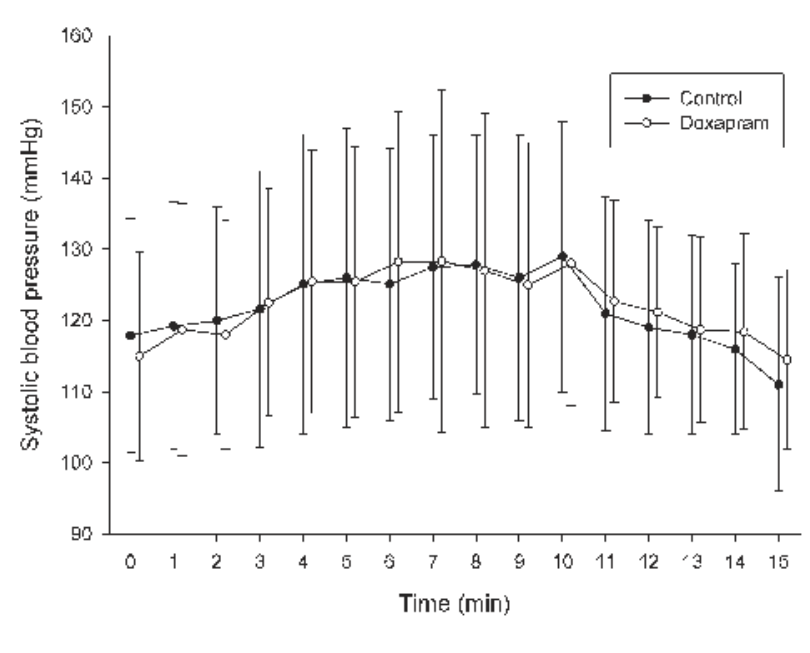

FIGURE 3 Changes in systolic blood pressure after injection of study drug.

minutes post-drug administration $(P<0.05)$. Beyond nine minutes, there were no differences in mean BIS scores between groups (Figure 1).

Following injection of study medication doxapram was associated with a significantly higher heart rate compared with the placebo group three to four minutes post-injection $(P<0.05$, Figure 2$)$. Changes in systolic blood pressure were similar in the two groups during emergence from anesthesia (Figure 3).

Aldrete recovery scores were similar in the two groups and all patients were discharged from PACU 
uneventfully, and fully awake. No patient experienced recall or awareness during anesthesia, or any unpleasant feeling upon awakening. No adverse effects were observed. Specifically, excitation, hallucinations, and anxiety reactions were not observed in any patient.

\section{Discussion}

The main finding of this study is that doxapram 1 $\mathrm{mg} \cdot \mathrm{kg}^{-1}$ iv hastens early recovery from sevoflurane anesthesia, and is associated with more rapid recovery of BIS values.

Doxapram is a central and peripheral respiratory stimulant and a nonspecific CNS stimulant. It is presently approved for the treatment of acute respiratory failure, postoperative respiratory depression, neonatal apnea of prematurity, and postanesthetic shivering. In the 1970s and up to the 1980s doxapram was not infrequently used as an analeptic antagonizing the hypnotic and/or respiratory depressant effects of sedatives such as diazepam, opioids, barbiturates and inhalation anesthetics commonly used at that time. ${ }^{10,14,15}$ With the introduction of selective antagonist drugs it is now well recognized that antagonism of opioids should be achieved with either naloxone or naltrexone, and antagonism of benzodiazepines can be achieved with flumazenil. However, there is a paucity of useful agents with reasonable safety profiles to antagonize the CNS depressant effects of inhalational anesthetics. Physostigmine has been used in the past, but this drug is now no longer available.

Recently, Turan et al. demonstrated that aminophylline $5 \mathrm{mg} \cdot \mathrm{kg}^{-1}$ iv hastens recovery from sevoflurane anesthesia. ${ }^{16}$ Sevoflurane, with a blood/gas coefficient of 0.65 , has a rapid induction time and a generally rapid recovery. ${ }^{17}$ However, slow recovery following sevoflurane is observed occasionally, and a pharmacological means to hasten recovery in such circumstances, without side effects, might be desirable and clinically useful. It therefore seemed logical to evaluate whether recovery from an inhaled anesthetic with a rapid recovery profile can be modified by a CNS stimulant such as doxapram, and whether BIS values correlate with the observed clinical response ${ }^{18,19}$ Noe et al. observed electroencephalogram (EEG) changes indicative of arousal with intraoperative administration of doxapram $0.5 \mathrm{mg} \cdot \mathrm{kg}^{-1}$ iv at the end of surgery under thiamylal or halothane- $\mathrm{N}_{2} \mathrm{O}$ anesthesia. However, these investigators did not elaborate on the specific EEG patterns. ${ }^{20}$ Roy and Stullken reported conversion of the EEG to an awake pattern in dogs under steady state halothane anesthesia, within $22 \pm$ $3 \mathrm{sec}$ following administration of doxapram $1 \mathrm{mg} \cdot \mathrm{kg}^{-1}$ $i{ }^{21}{ }^{21}$ In our study we found that doxapram $1 \mathrm{mg} \cdot \mathrm{kg}^{-1}$ $i v$ hastened recovery time in patients receiving sevoflurane anesthesia, and this improvement was also reflected in higher BIS scores. The arousal effect of doxapram appears to be directly related to its CNS stimulating effect, since both the end-tidal $\mathrm{CO}_{2}$ and the end-expiratory concentrations of sevoflurane were similar between groups. The observed changes in BIS values, attaining a significant between-groups difference within seven minutes, coincided with clinical signs of awakening. There were no episodes of recall/awareness or any other psychological reactions possibly related to the administration of doxapram. Of equal importance, there was no "re-narcotization" effect after doxapram's response had dissipated. Aldrete scores in the PACU showed no difference between the doxapram and placebo groups, and all the patients were discharged uneventfully.

A number of adverse effects have been reported with the use of doxapram, most noticeably tachycardia, cardiac arrhythmia, hypertension, hallucinations, excitation, anxiety reactions, and even panic attacks. ${ }^{7,9,10}$ More recently, Rosenberg et al. described the response of a doxapram infusion in an elderly patient following a laparotomy. The patient suffered a cerebrovascular accident, which the authors postulated may have been related to doxapram administration. ${ }^{22}$ In an animal study, Uehara $e t$ al. demonstrated that a large dose of doxapram $\left(50 \mathrm{mg} \cdot \mathrm{kg}^{-1}\right)$ given prior to bilateral carotid artery occlusion accentuates white matter damage in the neonatal rat. ${ }^{23}$ This seems to indicate that doxapram in large doses may render the brain more vulnerable to ischemic damage. For our investigation, we elected to use a modest single dose of doxapram $1 \mathrm{mg} \cdot \mathrm{kg}^{-1}$ which had previously been shown to be effective in reversing anesthetic effects, without adverse responses. ${ }^{7-10}$ However, benefits must always be weighed against potential risks, and we would only consider using small doses of doxapram in patients where recovery from inhalational anesthesia is unexpectedly prolonged.

The clinical significance of our observations must be considered in light of that fact that doxapram is no longer commonly used. We believe that our study provides clinically relevant information regarding the correlation between the BIS and the CNS arousal effect of a CNS stimulant. The BIS has been shown to be a useful guide in monitoring anesthetic recovery without drug intervention. ${ }^{18,19}$ and we have been able to demonstrate that BIS reflects changes during recovery from anesthesia following administration of an analeptic drug.

In conclusion, we have shown that administration of doxapram $1 \mathrm{mg} \cdot \mathrm{kg}^{-1} i v$ hastens early recovery 
from sevoflurane anesthesia, without appreciable side effects. The more rapid emergence correlates with higher BIS values when compared to placebo.

\section{References}

1 Moser KM, Luchsinger PC, Adamson JS, et al.

Respiratory stimulation with intravenous doxapram in respiratory failure. A double-blind co-operative study. N Engl J Med 1973; 288: 427-31.

2 Kato H, Buckly JP. Possible sites of action of the respiratory stimulant effect of doxapram hydrochloride. J Pharmacol Exp Ther 1964; 144: 260-4.

3 Hirsch K, Wang SC. Selective respiratory stimulating action of doxapram compared to pentylenetrorazaol. J Pharmacol Exp Ther 1974; 189: 1-11.

4 Mitchell RA, Herbert DA. Potencies of doxapram and hypoxia in stimulating carotid-body chemoreceptors and ventilation in anesthetized cats. Anesthesiology 1975; 42: 559-66.

5 Scott RM, Whitwan JG, Chakrabarti MK. Evidence of a role for the peripheral chemoreceptors in the ventilatory response to doxapram in man. Br J Anaesth 1977; 49: 227-31.

6 Po BT, Watson RL, Hansen HR. Arousal time following intravenous anesthetic agents, methohexital and thiopental: effect of doxapram hydrochloride. Anesth Analg 1968; 47: 446-51.

7 Siker ES, Mustafa K, Wolfson B. The analeptic effects of doxapram hydrochloride on thiopentone induced depression. Br J Anaesth 1964; 36: 216-23.

8 Gupta PK, Dundee JW. Hastening of arousal after general anaesthesia with doxapram hydrochloride. Br J Anaesth 1973; 45: 493-6.

9 Riddle PL, Robertson GS. Use of doxapram as an arousal agent in outpatient general anaesthesia. Br J Anaesth 1978; 50: 921-4.

10 Stephen CR, Talton I. Investigation of doxapram as a postanesthetic respiratory stimulant. Anesth Analg 1964; 43: 628-40.

11 Johansen JW, Sebel PS. Development and clinical application of electroencephalographic bispectrum monitoring. Anesthesiology 2000; 93: 1336-44.

12 Rosow C, Manberg PJ. Bispectral index monitoring. Anesthesiol Clin North America 2001; 19: 947-66.

13 Aldrete JA, Kroulik D. A postanesthetic recovery score. Anesth Analg 1970; 49: 924-34.

14 Ramamurthy S, Steen NS, Winnie AP. Doxapram antagonism of meperidine-induced respiratory depression. Anesth Analg 1975; 54: 352-6.

15 Allen CJ, Gough KR. Effect of doxapram on heavy sedation produced by intravenous diazepam. Br Med J (Clin Res Ed) 1983; 286: 1181-2.

16 Turan A, Memis D, Karamanlyoglu B, Colak A,
Pamukcu Z, Turan N. Effect of aminophylline on recovery from sevoflurane anaesthesia. Eur J Anaesthesiol 2002; 19: 452-4.

17 Miller RD. Anesthesia, 5th ed. Philadelphia, PA: Churchill Livingstone; 2000: 2226-7.

18 Anderson RE, Barr G, Assareh H, Jakobsson J. The AAI index, the BIS index and end-tidal concentration during wash in and wash out of sevoflurane. Anaesthesia 2003; 58: 531-5.

19 Muncaster AR, Sleigh JW, Williams M. Changes in consciousness, conceptual memory, and quantitative electroencephalographical measures during recovery from sevoflurane- and remifentanil-based anesthesia. Anesth Analg 2003; 96: 720-5.

20 Noe FM, Borrillo N, Greifenstein FE. Use of a new analeptic, doxapram hydrochloride, during general anesthesia and recovery. Anesth Analg 1965; 44: 206-13.

21 Roy RC, Stullken EH. Electroencephalographic evidence of arousal in dogs from halothane after doxapram, physostigmine, or naloxone. Anesthesiology 1981; 55: 392-7.

22 Rosenberg J, Kristensen PA, Pedersen MH, Overgaard $H$. Adverse events with continuous doxapram infusion against late postoperative hypoxaemia. Eur J Clin Pharmacol 1996; 50: 191-4.

23 Uehara H, Yoshioka $H$, Nagai $H$, et al. Doxapram accentuates white matter injury in neonatal rats following bilateral carotid artery occlusion. Neurosci Lett 2000; 281: 191-4. 